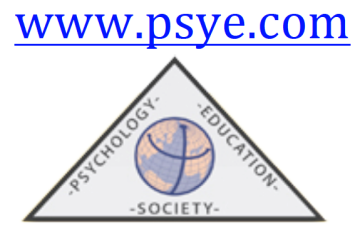

\title{
La dimensión intercultural en la formación docente en Ecuador
}

\author{
Enrique ESPINOZA FREIRE ${ }^{1}$, Luis Alberto HERRERA MONTERO ${ }^{2}$ y \\ José Manuel CASTELLANO GIL ${ }^{3}$ \\ ${ }^{1}$ Universidad Técnica de Machala, Ecuador \\ ${ }^{2}$ Universidad de Cuenca, Ecuador \\ ${ }^{3}$ Universidad Tecnológica Indoamérica, Ecuador
}

(Recibido el 4 de Enero de 2019, Aceptado el 5 de Septiembre de 2019)

\begin{abstract}
RESUMEN: Este artículo tiene como objeto caracterizar la dimensión intercultural del proceso de formación docente en el estudiantado que cursa la carrera de Educación Básica en la Universidad Técnica de Machala (UTMach) en Ecuador durante el período académico 2016-2017. La estrategia metodológica responde a un paradigma mixto, cuantitativo-cualitativo, sistematizada mediante los métodos de análisis documental, observación científica, estadístico y triangulación de datos. Los resultados evidencian la inexistencia de contextualización de esa dimensión en el currículum, insuficientes metodologías para su tratamiento, actitudes estudiantiles tipificadas de "muy mejorables" y "mejorables" desde una perspectiva axiológica al no reconocer cabalmente los derechos étnicos y culturales de todos los ciudadanos, particularmente entre los alumnos de primer y segundo año. Por tanto, a la luz de estos datos se requiere activar un diseño y redirección estratégica en el ámbito de la formación intercultural.
\end{abstract}

Palabras claves: Interculturalidad; legislación educativa; diversidad cultural; currículum; actitudes estudiantiles.

\section{The intercultural dimension in the teaching formation in Ecuador}

\begin{abstract}
The purpose of this article is to characterize the intercultural dimension of the in the teaching formation process in the student body that studies the Basic Education course in the Technical University of Machala (UTMach) in Ecuador during the 2016-2017 academic period. The methodological strategy responds to a mixed, quantitative-qualitative paradigm, systematized through the methods of documentary analysis, scientific observation, statistics and data triangulation. The results show the lack of contextualization of this dimension in the curriculum, insufficient methodologies for its treatment, typified student attitudes of "very improvable" and "improvable" from an axiological perspective by not fully recognizing the ethnic and cultural rights of all citizens, particularly among first and second year students. Therefore, in light of these data it is necessary to activate a design and strategic redirection in the field of intercultural formation.
\end{abstract}

Keyword: Interculturality; educational legislation; cultural diversity; curriculum; student attitudes. 
Correspondencia: Enrique Espinoza Freire. Universidad Técnica de Machala, Av. Panamericana Km. 5 1/2 Vía a Pasaje, Machala, Ecuador. E-mail: jubonista15@hotmail.com

\section{Introducción}

Ecuador es un país plurinacional e intercultural, cuya población se caracteriza por la heterogeneidad étnica, constituida fundamentalmente por indígenas, blancos, negros y mestizos, y por una diversidad de lenguas y culturas (Conejo-Arellano, 2008), una condición que debe ser preservada dentro de un contexto mundial globalizado. En tal sentido, el Gobierno y el Estado ecuatoriano, en las últimas décadas han realizado esfuerzos encaminados a garantizar la salvaguarda de lo que constituye una de las principales riquezas culturales de la nación.

En ese empeño se desarrollan políticas públicas que legitiman la no discriminación de los ciudadanos por color de la piel, cultura, credos o zona geográfica de residencia. Dentro de esas políticas se encuentran las relativas a la actividad educacional que tienen como base legal la Constitución de la República del Ecuador (2008). En tal sentido en el artículo 38 se declara que el Estado al instituir estas políticas tendrá en consideración las diferencias específicas entre las etnias, culturas, comunidades, pueblos y nacionalidades; en particular se tomarán medidas que garanticen la educación en un marco de protección integral de derechos.

En correspondencia en el artículo 57 se establece que se debe desarrollar, fortalecer y potenciar el sistema de educación bilingüe en todos los niveles de enseñanza, conforme a la diversidad cultural para el cuidado y preservación de las identidades en consonancia con sus metodologías de enseñanza y aprendizaje. Contribuyendo a garantizar la dignidad y diversidad de sus culturas, tradiciones, historia y aspiraciones reflejadas en la educación pública. De igual forma el artículo 347 establece la garantía del sistema de educación intercultural bilingüe, en el cual se ha de utilizar como lengua principal de educación la de la nacionalidad respectiva y el castellano como idioma de relación intercultural, así como el acceso a la educación pública bajo los principios de equidad social, territorial y regional.

En respuesta a estas normativas el Ministerio de Educación de la República del Ecuador busca alternativas para mejorar los niveles de instrucción y educación de la población que reciben sus ciudadanos, en consecuencia, implementa políticas educativas al amparo de este marco legal (Treviño, Villalobos \& Baeza, 2016; UNESCO, 2016). En tal sentido se promulga como instrumento normativo y político la Ley Orgánica de Educación Intercultural (LOEI) en el 2011, que reitera la importancia y necesidad de crear espacios de diálogo entre conocimientos y saberes tradicionales (Krainer, Aguirre, Guerra y Meise (2017), para de manera creativa vincular las disímiles formas de conocimiento más allá de las fronteras culturales (Almeida, 2014).

En la Ley Orgánica de Educación Intercultural se explicita que en el Ecuador existe el derecho a la educación gratuita sin discriminación alguna desde el nivel Inicial hasta el Bachillerato; previendo además los derechos de la población rural e indígena a estos servicios. En la mencionada Ley, en su artículo 2, se establecen los principios de la actividad educativa, entre ellos el de la universalidad, que plantea que la educación es un derecho humano fundamental y que es deber inexcusable del Estado garantizar el acceso, permanencia y calidad de la educación para toda la población sin ningún tipo de discriminación. También, contempla el principio de la educación en valores, que ha de basarse en la transmisión y práctica de (c) Psy, Soc, \& Educ, 2019, Vol. 11(3) 
valores que promuevan la libertad personal, el respeto a los derechos, a la democracia y a la diversidad étnica y religiosa, la igualdad y la eliminación de toda forma de discriminación, garantizando así las manifestaciones culturales diversas sin segregación de ningún tipo.

Por su parte el principio de enfoque de derecho complementa el anterior al declarar que la educación deberá incluir el conocimiento de los derechos, sus mecanismos de protección y exigibilidad, ejercicio responsable, reconocimiento y respeto a las diversidades, en un marco de libertad, dignidad, equidad social y cultura (LOEI, 2011, p. 9).

Por otra parte, el principio de comunidad de aprendizaje considera la educación como espacio de diálogo social e intercultural, de igual forma el principio de equidad e inclusión garantiza el respeto a la diversidad de culturas en la teoría y en la práctica.

$\mathrm{La}$ interculturalidad y plurinacionalidad, como principio que garantiza el reconocimiento, respeto y valoración de las diferentes nacionalidades, culturas y pueblos que conforman el Ecuador, así como sus saberes ancestrales propugnando la unidad en la diversidad, propiciando el diálogo intercultural e intracultural, y propendiendo a la valoración de las formas y usos de las diferentes culturas que sean consonantes con los derechos humanos. Mediante el principio de identidades culturales se garantiza al ciudadano el derecho a una educación que le permita construir y desarrollar su propia identidad cultural y su libertad de elección y adscripción identitaria, aportando espacios de reflexión, visibilización, fortalecimiento y el robustecimiento de su cultura.

A través del plurilingüismo se reconoce el derecho a todas las personas, comunas, comunidades, pueblos y nacionalidades a formarse en su propia lengua como reconocimiento a sus derechos culturales y en idiomas oficiales de relación intercultural.

En el principio de unicidad y apertura, se plantea que el sistema de educación es único y reconoce la diversidad de la sociedad ecuatoriana, intercultural y plurinacional. Por último, el principio de pertinencia aboga por una formación que responda a las necesidades del entorno social, natural y cultural.

En concordancia con estas normativas, legislaciones y principios el Consejo de Educación Superior (2017), en el artículo 3 del Reglamento de Régimen Académico, declara entre sus objetivos contribuir al desarrollo de profesionales comprometidos con las transformaciones de los entornos sociales y naturales, respetando la interculturalidad, igualdad de género y demás derechos constitucionales (p. 3).

En correspondencia en los artículos 50 y 51 se aborda la articulación de la interculturalidad con los diferentes campos formativos. En el artículo 50 se indica que el currículo debe incorporar criterios de interculturalidad en cada nivel de formación, organización curricular y campo formativo. Legisla que esta incorporación se podrá realizar de las siguientes formas:

a) Modelos de aprendizaje. Contextualización de los aprendizajes a través de metodologías educativas que promuevan el reconocimiento de la diversidad cultural y el diálogo de saberes. Desarrollará la referencia a conocimientos pertenecientes a diversas cosmovisiones, epistemologías o perspectivas de pueblos, nacionalidades o grupos socioculturales

b) Itinerarios académicos. Creación de asignaturas y cursos o itinerarios específicos dentro de una carrera o programa académico, que integren saberes ancestrales y de 
aplicación práctica en determinados campos de formación profesional, siempre que se garantice su coherencia y pertinencia

c) Modelos interculturales de educación superior. Generación de modelos educativos interculturales integrales, a través del diseño e implementación de carreras, programas o la creación de instituciones de educación superior o de sus unidades académicas, específicas para estos fines (Consejo de Educación Superior, 2017, p. 23).

Asimismo, el artículo 51, indica las estrategias para lograr esta articulación con los diferentes tipos de carrera de formación:

a) Incorporar en los contenidos curriculares los saberes, enfoques, tecnologías y prácticas de los pueblos, nacionalidades y otros grupos socioculturales.

b) Adaptar la formación académica al contexto socio cultural y territorial de los pueblos y nacionalidades indígenas, utilizando como medio de aprendizaje las lenguas nativas correspondientes.

c) Desarrollar carreras de educación intercultural bilingüe (Consejo de Educación Superior, 2017, p. 23).

Como parte de este empeño y cambios se enmarca la educación intercultural para todos y en los diferentes niveles, establecida en el Ecuador en la Constitución del año 2008, tiene su origen en la Educación Intercultural Bilingüe, como fruto de la labor reivindicativa de los pueblos originarios; importante propuesta educativa que responde a los requerimientos políticos, filosóficos, culturales y lingüísticos de las diferentes culturas en aras de lograr una mejor calidad educativa y de vida de esa población (Krainer, 2010).

La Constitución expresa las ideas del "Buen Vivir" como facilitadoras de cambios estructurales en el país, anclados en un nuevo sistema de educación que tiene como centro el aprendiz con una visión intercultural acorde a la diversidad geográfica, cultural y lingüística, en un marco de respeto a los derechos (Asamblea Nacional del Ecuador, 2008; Krainer \& Guerra, 2016).

De esta forma la nueva función docente queda marcada por una cierta intensificación de las tareas que no se ajustan plenamente a las expectativas previstas durante su período formativo (Hargreaves, 2005; Bolívar, 2007), la labor docente ha tenido que ser redireccionada experimentando importantes cambios; debiendo asumir nuevas tareas y responsabilidades.

Son muchos los estudiosos del tema de la interculturalidad en los espacios de formación docente, tanto nacionales como extranjeros, entre ellos se destacan por su significación para este estudio los trabajos de Rodríguez (2005), Aguado, Gil y Mata (2008), Rodríguez y Hernández (2010), Viaña (2011), Bello-Domínguez (2013), Santos, Albulhamail et al. (2014), Cernadas y Lorenzo (2014), Elisondo y Donolo (2014), Flores, Prat y Soler (2014), Villagómez y Cunha de Campos (2014), Uribe-Pérez y Mosquera (2016) y Krainer et al. (2017); quienes demuestran que las actitudes de los docentes hacia la diversidad cultural se correlacionan altamente con su nivel formativo; se necesitan experiencias formativas para desarrollar una comunidad educativa bien informada y capaz de la inclusión desde una mirada de la diversidad de culturas.

Por otro lado, aún algunos currículos presentan limitaciones en el orden socio-cultural, entre estas falencias cobra trascendencia el tratamiento desde lo general de las características singulares de lo indígena, lo que obstaculiza su plena inserción en el ámbito educacional y el 
rendimiento académico de la población estudiantil originaria; por otra parte, muchos de los maestros asignados a estas comunidades no conocen sus costumbres, tradiciones, lengua, cultura y realidades (Krainer et al, p. 1).

Esta praxis educativa que durante mucho tiempo se ha brindado a los pueblos indígenas, ha ocasionado su asimilación de manera indiscriminada, contribuyendo a limitar su desarrollo socio-cultural y económico, provocando así la fractura de su identidad y manifestaciones de racismo (Conejo-Arellano, 2008, p. 2). Ello evidencia que, más allá del discurso, de normas y legislaciones legales, la interculturalidad es aún un concepto por comprender, "que requiere de la reflexión crítica y voluntad política para establecerse como principio real de una sociedad diversa" (Krainer, et al. 2017, p. 1).

Contribuciones como las Raúl Fornet Betancour (2009) definen por interculturalidad a procesos de descolonización, donde el poder se comparta a partir de nuevas manifestaciones de subjetivación. Por su parte, Fidel Tubino (2004), concibe que la interculturalidad es más una práctica que una teoría, es un pacto ético de respeto entre pluralidad de culturas. En definitiva, la interculturalidad son procesos que superan las limitaciones de los sistemas nacionales, ya que promueve la concreción de sistemas plurinacionales y cosmopolitas (Herrera, 2015).

En términos de educación, el tema intercultural ha sido trabajado, principalmente, en América Latina. Ruth Moya (1998) comparte una síntesis de la historia de la EIB, desde sus componentes políticos y jurídicos. Otros autores, como Luis Enrique López (2009) realiza análisis sobre la EIB en relación a la lucha de los pueblos indígenas en América Latina; sus conquistas en términos de descolonización, reforzamiento de su identidad y posicionamiento de su dignidad política. En un intento por articular también a otros pueblos, Sabine Speicer (1999) considera lo siguiente: "es un principio que va mucho más allá del sector educativo y apunta al desarrollo de sociedades no solo multiétnicas y pluriculturales por su composición poblacional sino interculturales por sus principios de participación y respeto mutuo" (p. 85). Cabe entonces la caracterización de la dimensión intercultural mediante el análisis reflexivo en torno a las estrategias asumidas por el claustro de profesores de la carrera de Educación Básica de la UTMach para la formación de docentes y la importancia que ocupa al respecto la policromía cultural en la educación de ciudadanos con un fin común, el desarrollo sostenible y el avance del país hacia una sociedad más justa y equitativa.

\section{Metodología}

La estrategia metodológica de la investigación responde a un paradigma mixto, cuantitativo-cualitativo, sistematizada a través de los métodos de análisis documental, observación científica, estadístico y triangulación de datos.

El análisis documental permitió la construcción epistémica de los referentes teóricos, que junto a la observación científica facilitaron la determinación de las regularidades del proceso de formación docente con énfasis en la dimensión intercultural. A través del método estadístico se planificó, recolectó, procesó y analizó la información obtenida mediante la técnica del cuestionario. La triangulación de los datos recolectados mediante los diferentes métodos garantizó la validez y fiabilidad de los resultados.

\section{Participantes}


La población estuvo constituida por 229 estudiantes de la carrera de Educación Básica de la UTMach durante el período académico 2016-2017.

\section{Instrumentos}

La observación científica se desarrolló a través de una guía de observación científica para caracterizar la dimensión intercultural del proceso de formación docente; los parámetros tenidos en consideración fueron:

- Contextualización del currículum a la realidad intercultural del país

- Presencia de los principios de la actividad educativa relativos a la dimensión intercultural

- Metodologías educativas para promover el reconocimiento de la diversidad cultural

- Presencia de estrategias de integración cultural en el proceso de enseñanzaaprendizaje

Se aplicó la técnica del cuestionario a los futuros docentes acerca de las percepciones sobre la atención a la dimensión intercultural durante la formación. Se empleó una variante del cuestionario validado por Rodríguez y Hernández (2010) en el estudio "Cuestionario de Actitudes hacia la Multiculturalidad-CAM", adaptado al contexto y realidad ecuatoriana; fue estructurado en tres apéndices con el objetivo de determinar los posibles prejuicios racistas y culturales del alumnado y establecer la percepción de los estudiantes sobre el tratamiento a la dimensión intercultural en el proceso de formación.

A cada pregunta el alumnado tenía que responder con un valor numérico entre 0 (totalmente en desacuerdo) y 4 (totalmente de acuerdo). Una vez concluido este proceso, se clasificó la actitud de los estudiantes ante los prejuicios: raciales y culturales; así como su percepción sobre el tratamiento pedagógico de la dimensión intercultural, otorgándoles la categoría de "muy mejorable", "mejorable", "aceptable" o "bueno"; lo que facilitó la caracterización de este proceso y la proyección, así como el direccionamiento de estrategias que permitan actuar de manera más específica.

Los ítems que conforman cada apéndice fueron los siguientes:

Apéndice-A. Prejuicios racistas

1) Ninguna raza tiene el patrimonio de la belleza

2) La raza no tiene relación con la inteligencia

3) Un hombre o mujer de cualquier raza puede obtener una medalla de oro en cualquier deporte

4) Algunas razas huelen peor que otras

5) Aceptaría tener una relación afectiva con alguien de otra raza o cultura

6) Los indígenas tienen más enfermedades

7) Si mis padres adoptaran un niño, no me importaría que fuera de otra raza

8) Hay razas mejores que otras en general

Apéndice-B. Prejuicios culturales

1) La emigración a las ciudades aumenta la delincuencia de forma desproporcionada

2) Todas las culturas son igualmente respetables

3) La cultura occidental es superior a todas las demás

4) En el Ecuador sólo se debería hablar en español

5) Los indígenas tienen derecho a hablar su dialecto entre ellos en Ecuador

6) Todas las vestimentas son aceptables 
7) Los indígenas deberían renunciar a sus costumbres si quieren vivir en las ciudades

8) Los indígenas tienen el derecho y el deber de aprender español

9) Todas las culturas enriquecen la idiosincrasia cultural

10) Una sociedad culta y desarrollada debe ser acogedora y tolerante

11) Entre los diferentes pueblos que conforman la nación hay más semejanzas que diferencias y es más lo que nos une que lo que nos separa

12) Debemos admitir y respetar todas las costumbres y tradiciones

13) La fusión y el mestizaje son fuentes de riquezas

Apéndice-C. Percepción del tratamiento pedagógico de la interculturalidad

1) Contextualización del currículum a la realidad intercultural del país

2) Presencia de los principios de la actividad educativa relativos a la dimensión intercultural

3) Metodologías educativas para promover el reconocimiento de la diversidad cultural

4) Presencia de estrategias de integración cultural en el proceso de enseñanzaaprendizaje

La interpretación de los resultados se realizó atendiendo a la puntuación total acumulada en cada apéndice, tal como se muestra en la tabla 1.

Tabla1. Actitudes hacia la dimensión multiculturalidad-CAM

\begin{tabular}{|c|c|c|c|c|}
\hline \multirow[t]{2}{*}{ Actitud } & \multicolumn{3}{|c|}{ Apéndices } & \multirow[t]{2}{*}{ Puntuación total } \\
\hline & $\mathrm{A}$ & $\mathrm{B}$ & $\mathrm{C}$ & \\
\hline Muy mejorable & Entre 0 y 8 puntos & Entre 0 y 13 puntos & Entre 0 y 4 puntos & Entre 0 y 25 puntos \\
\hline Mejorable & Entre 9 y 16 puntos & Entre 14 y 26 puntos & Entre 5 y 8 puntos & Entre 26 y 50 puntos \\
\hline Aceptable & Entre 17 y 24 puntos & Entre 27 y 39 puntos & Entre 9 y 12 puntos & Entre 51 y 75 puntos \\
\hline Buena & Entre 25 y 32 puntos & Entre 40 y 52 puntos & Entre 13 y 16 puntos & Entre 76 y 100 puntos \\
\hline
\end{tabular}

\section{Procedimiento}

La investigación contó con tres fases o momentos; en una primera se procedió a la preparación teórico-metodológica de los miembros del equipo de investigación y la confección de los instrumentos para la recogida de la información. En un segundo momento se aplicó el instrumento para lo cual se designó un grupo de tres profesores por cada año académico. En la tercera fase se procesó y analizó la información recolectada por cada uno de los grupos formados; una vez concluido este análisis se integraron los equipos para el cotejo y análisis general de la información obtenida a través de los diferentes métodos empleados.

\section{Análisis de resultados}

Los datos se procesaron a través de paquete estadístico SPSS versión 15.0, se determinaron las frecuencias absolutas y relativas las que se resumieron en tablas descriptivas.

\section{Resultados y Discusión}

\section{Resultados y discusión de la observación científica}


A través de la observación científica y directa sobre 45 actividades docentes del proceso de formación, se pudo determinar que:

Aún existen limitaciones en la contextualización de los currículum al tratamiento de la interculturalidad en el proceso de formación docente; los programas de asignaturas y disciplinas en el $60 \%$ (27) de los casos no explicitan cómo proceder para dar salida a esta dimensión.

A pesar de ello, se pudo constatar que, en el 86,7\% (39) de las actividades observadas, el docente de una manera intencional tiene en cuenta los principios de la actividad educativa relacionados con la dimensión intercultural: universalidad, educación en valores, enfoque de derecho, comunidad de aprendizaje, equidad e inclusión, interculturalidad y plurinacionalidad, identidades culturales, unicidad y apertura, plurilingüismo y pertinencia; destacando la necesidad de establecer en la praxis educativa el reconocimiento y mecanismos que garanticen el conocimiento del marco legal que ampara la diversidad de culturas; así como la protección, respecto, libertad equidad social de todas las nacionalidades, culturas y pueblos que conforman la nación ecuatoriana. Asimismo, se trasmite y desarrolla en los estudiantes el respeto a la concepción única del sistema educativo con carácter intercultural y plurinacional en correspondencia con el entorno social, natural y cultural (LOEI, 2011; Treviño et al. 2016).

Además, se establece que la educación debe ser un espacio intercultural donde prime el diálogo reflexivo en el respeto a las tradiciones y costumbres de todos los pueblos; también se trabaja con los futuros docentes en el desarrollo de valores que garanticen los derechos de las diferentes nacionalidades, culturas y pueblos, destacando el que poseen a una educación que lejos de adaptarlos a las costumbres occidentales, les sirva de herramienta para desarrollar su propia identidad cultural y el respeto a su lengua originaria (Consejo de Educación Superior, 2017).

Si bien no existe una metodología establecida en los currículos para promover el reconocimiento de la diversidad cultural, cada asignatura desde un enfoque práctico metodológico aborda el tema en correspondencia al sistema de contenidos de cada ciencia en particular. Lo que se pudo apreciar en el 68,9\% (31) de las observaciones. En correspondencia en el 73,3 (33) de estas actividades docentes se aprecia la presencia de estrategias educativas encaminadas a mostrar el cómo lograr la integración cultural en el contexto del proceso de enseñanza-aprendizaje. A través de éstas los docentes contribuyen a reducir la asimetría entre las normativas y las limitaciones prácticas de los currículos.

En términos específicos, estos datos facultan la necesidad institucional de diagnosticar la apertura de autoridades, docentes, estudiantes y la comunidad educativa en su conjunto, para fortalecer procesos pluriliguísticos, que implique al menos la integración del kichwa o el shuar, como lenguas originarias de mayor presencia en el ámbito nacional, en los procesos de aprendizaje y diseño curricular de la oferta académica universitaria, particularmente en la carrera de Educación General Básica. De igual manera, que las distintas carreras puedan articular los saberes ancestrales como iniciativa de fomento académico profesional, basada en el respectivo diálogo epistémico que se promueve, principalmente en materia de educación, como principio constitucional. Así se lograría no solamente generar una actitud positiva de la comunidad educativa, sino también procesos con niveles de concreción.

La sugerencia recientemente descrita, como iniciativa que surge del análisis de datos, requiere de procesos integrales de desaprendizaje, motivación y nueva formación en el personal 
docente, con el propósito de implementar políticas y procesos sostenidos en el diálogo epistémico y en la práctica educativa intercultural, como se lo determina en la Constitución de 2008.

A pesar de las insuficiencias observadas existen acciones direccionadas a lograr en los estudiantes en formación el desarrollo de valores que permitan el adecuado tratamiento de la interculturalidad en el futuro ejercicio de su profesión; además se evidencia el interés por trasmitirles experiencias, procedimientos y vías que contribuyan desde lo pedagógico a este empeño.

\section{Resultados y discusión del cuestionario}

Los resultados obtenidos mediante el cuestionario aplicado a los 229 estudiantes se resumen en la siguiente tabla.

Tabla 2. Actitudes hacia la dimensión interculturalidad de los estudiantes de la Carrera de Educación Básica de la UTMach durante el período académico 2016-2017.

\begin{tabular}{lcccccccc}
\hline \multicolumn{1}{c}{ Actitud } & \multicolumn{2}{c}{$\begin{array}{c}\text { Prejuicios } \\
\text { racistas }\end{array}$} & \multicolumn{2}{c}{$\begin{array}{c}\text { Prejuicios } \\
\text { culturales }\end{array}$} & \multicolumn{2}{c}{$\begin{array}{c}\text { Dimensión } \\
\text { intercultural }\end{array}$} & \multicolumn{2}{c}{$\begin{array}{c}\text { Evaluación } \\
\text { General }\end{array}$} \\
\cline { 2 - 10 } & Frecuencia & $\%$ & Frecuencia & $\%$ & Frecuencia & $\%$ & Frecuencia & $\%$ \\
\hline Muy mejorable & 59 & 17.9 & 65 & 19.8 & 56 & 17.0 & 60 & 18.2 \\
\hline Mejorable & 98 & 29.8 & 84 & 25.5 & 79 & 24.0 & 77 & 23.4 \\
\hline Aceptable & 104 & 31.6 & 98 & 29.8 & 105 & 31.9 & 104 & 31.6 \\
\hline Buena & 68 & 20.7 & 82 & 24.9 & 89 & 27.1 & 88 & 26.7 \\
\hline
\end{tabular}

En las acciones realizadas al interior del proceso de formación de docentes para la Educación Básica aún se evidencian insuficientes. En general el 42\% de los estudiantes presentan actitudes que pueden ser "muy mejorables" o "mejorables" desde el punto de vista axiológico para superar sus prejuicios raciales y culturales; así como incorporar la dimensión intercultural en su repertorio de acciones profesionales.

Es significativo señalar que estas manifestaciones se dan particularmente en los estudiantes del primer y segundo años de la carrera; existe tendencia hacia actitudes aceptables y buenas a partir del 3er año; lo que puede ser entendido como un proceso de maduración y el resultado de la labor de los docentes. Por lo que las estrategias han de ser direccionadas con mayor intencionalidad a los alumnos del primer y segundo cursos. Situación similar encontrada por Rodríguez y Hernández (2010) en sus estudios, donde los alumnos de menor graduación manifestaron actitudes negativas especialmente en el acápite de prejuicios racistas.

Se aprecia correspondencia entre la actitud de los estudiantes y la percepción del tratamiento de la dimensión intercultural (Flores et al., 2014; Krainer, 2016); lo que tiene su explicación en la relación que se establece entre el nivel formativo alcanzado y la visualización de la diversidad cultural (Albulhamail et al., 2014; Uribe-Pérez y Mosquera, 2016), donde mucho tiene que ver la formación de valores y la concepción de la interculturalidad como diálogo de saberes, y no como una mirada reduccionista de lo indígena (Olivera-Rodríguez, 2014; Krainer et al., 2017).

En consecuencia, es preciso establecer estrategias en la formación de valores y principios que permitan observar y respetar los derechos de estos pueblos. No obstante, la 
formación de valores implica procesos de reaprendizaje teórico, pero también la deconstrucción de prácticas estudiantiles, en torno a manifestaciones de índole racistas y claramente herederas de comportamientos coloniales. De esto modo, se aplicaría lo sostenido por Fidel Tubino (2004), en cuanto a concebir la interculturalidad como pacto estrictamente ético.

\section{Resultados y discusión del análisis documental}

El análisis documental además de permitir el estudio del marco legal de las políticas educativas en torno a la dimensión intercultural en la formación docente, facilitó el estudio y revisión de los currículos de la carrera. Se determinó que existen insuficiencias de orden formal en su contextualización; en los programas, en general, no se explicitan sus particularidades y procedimientos específicos, se da un tratamiento generalizador; sin embargo, a lo interno de las asignaturas y disciplina los docentes realizan la preparación metodológico para el tratamiento de esta dimensión, lo que se evidencia en la implementación de estrategias, aunque estás aún pueden ser perfeccionadas en la articulación de la interculturalidad a través de algunas propuestas del Consejo de Educación Superior (2017):

- Abordar, en los contenidos curriculares, los saberes correspondientes a los principales enfoques epistemológicos y perspectivas históricas de las nacionalidades y pueblos ancestrales, y otros grupos socio culturales, garantizando el diálogo intercultural de las ciencias.

- Estimular, en las carreras, perspectivas y saberes genuinamente interculturales.

- Propiciar el diálogo de saberes articulando el bagaje científico tecnológico, con las cosmovisiones, epistemologías y saberes prácticos, tradicionales, ancestrales y cotidianos, así como con el reconocimiento de las particularidades lingüísticas implicadas.

- Potenciar la diversidad y del aprendizaje intercultural, se propenderá a la implementación de ambientes, metodologías de aprendizaje, procesos y procedimientos que respeten y potencien la identidad étnica y las capacidades diversas.

Así como prever y priorizar la formación de maestros indígenas de las mismas comunidades, para que desarrollen su trabajo educativo utilizando la lengua materna, revalorizando así su cultura (Conejo-Arellano, 2008; Lehmann, 2015), contribuyendo al cumplimiento del artículo 347 de la Constitución.

Para el contexto particular de la carrera de Educación General Básica de Universidad Técnica de Machala (UTMach), si bien no es una propuesta disciplinar de educación intercultural bilingüe, es preciso comprender que la interculturalidad educativa integra a comunidades pueblos y nacionalidades, pero involucra también la diversidad poblacional identificada dentro del mestizaje. Entonces el diálogo epistémico y la práctica intercultural son procesos de desaprendizaje y reaprendizaje, que debe concretarse en forma integral en la planificación curricular, en el trabajo de aula, en los proyectos de investigación y en las prácticas preprofesionales.

La triangulación de los datos obtenidos mediante la aplicación de los métodos de observación científica, análisis documental y estadístico permitió determinar que existe 
congruencia en los resultados obtenidos por esas diversas vías, garantizando la validez y fiabilidad de las conclusiones.

\section{Conclusiones}

Los resultados obtenidos permiten caracterizar la dimensión intercultural del proceso de formación docente en la carrera de Educación Básica en la UTMach. Aunque existe una intencionalidad en las acciones de los profesores por lograr una formación en valores para el adecuado tratamiento de esta dimensión en el futuro ejercicio de la profesión; aún se evidencia su no contextualización en el currículum de las asignaturas y disciplinas, no se explicitan las metodologías particulares para su tratamiento en el proceso de formación docente; existen actitudes de los estudiantes tipificadas como "muy mejorables" y "mejorables" desde una perspectiva axiológica al no reconocer cabalmente los derechos raciales y culturales de todos los ciudadanos, particularmente entre los alumnos del primer y segundo años de la carrera.

Partiendo del reconocimiento de estas fisuras se deben perfeccionar y redireccionar las acciones para el desarrollo de actitudes no discriminatorias, que estime la integración intercultural y plurinacionalidad en el quehacer educativo, asegurando el reconocimiento y respeto a la diversidad de culturas, pueblos y nacionalidades que conforman el Ecuador. La educación intercultural, en el ámbito de la educación superior, debe concebirse y practicarse como procesos continuos de construcción, deconstrucción y reconstrucción, que valoren el hecho de educar desde la mutua convivencia, como aprendizaje para compartir el poder, como un pacto ético que se aprende, desaprende y reaprende en marcos igualdad de derechos y prácticas cosmopolitas.

La educación intercultural conlleva desafíos para Universidad Técnica de Machala (UTMach), con especificidad para la carrera de Educación General Básica. La iniciativa implica el fortalecimiento de acciones para implementar el diálogo epistémico y la práctica respetuosa de los derechos de comunidades, pueblos y nacionalidades, a través de tareas que faciliten el desaprendizaje de comportamientos racistas y coloniales, que requiere de esfuerzos de corresponsabilidad entre autoridades, docentes, estudiantes, iniciando con los participantes de esta investigación, y padres de familia, articulados todos como comunidad educativa.

\section{Referencias}

Aguado, M. T., Gil, I. y Mata, P. (2008). El Enfoque intercultural en la formación del profesorado: dilemas y propuestas. Revista Complutense de Educación, 2(19), 275-292. Disponible en https://papers.ssrn.com/sol3/papers.cfm?abstract_id=2147224

Albulhamail, S., Al-Sulami, F., Alnouri, M., Mahrous, N., Joharji, D., Albogami, M. et al. (2014). Primary school teacher's knowledge and attitudes towards children with epilepsy. Seizure European Journal of Epilepsy, 23(4), 280-283. Disponible en https://www.sciencedirect.com/science/article/pii/S105913111300352X

Almeida, I. (2014). Línea de Fuego. ¿Educación Intercultural Bilingüe para todos? Disponible en https://alineadefuego.info/2014/02/06/educacion-intercultural-bilingue-para-todospor-ileana-almeida/

Asamblea Nacional del Ecuador (2008). Constitución Política del Ecuador. Disponible en http://repositorio.dpe.gob.ec/bitstream/39000/638/1/NN-001-Constituci\%C3\%B3n.pdf 
Bello-Domínguez, J. (2013). Educación intercultural. ¿Trabajar con los diferentes o las diferencias? Universidad Autónoma Indígena de México El Fuerte, México. Ra Ximhai, 9(1), enero-abril, 61-73. Disponible en http://www.redalyc.org/articulo.oa?id=46126366009

Bolívar, A. (2007). La formación inicial del profesorado de secundaria y su identidad profesional. Estudios sobre Educación, 12, 13-30. Disponible en https://dadun.unav.edu/handle/10171/8987

Conejo-Arellano, A. (2008). Educación Intercultural Bilingüe en el Ecuador. La propuesta educativa y su proceso. Universidad Politécnica Salesiana Cuenca, Ecuador. Alteridad. Revista de Educación, 3(2), 64-82. Disponible en http://www.redalyc.org/articulo.oa?id=467746251005

Consejo de Educación Superior (25 de enero de 2017). Resolución del Consejo de Educación Superior 51. Reglamento de Régimen Académico Consejo Educación Superior. Registro Oficial Edición $\quad$ Especial, 854. Disponible en http://www.ces.gob.ec/lotaip/2018/Enero/Anexos\%20Procu/An-lit-a2Reglamento\%20de\%20R\%C3\%A9gimen\%20Acad\%C3\%A9mico.pdf

Elisondo, R. \& Donolo, D. (2014). Interculturalidad, apertura a experiencias y creatividad Aportes para una educación alternativa. RED, Revista de Educación a Distancia. 4(1). Disponible en http://www.um.es/ead/red/41

Fornet-Betancour, R. (2009). Interculturalidad en procesos de subjetivación. Coordinación General de Educación Intercultural y Bilingüe, México. Disponible en https://centroderecursos.cultura.pe/sites/default/files/rb/pdf/interculturalidad_betancourt.pdf

Flores, G., Prat, M. y Soler, S. (2014). La voz del profesorado de Educación Física sobre su formación académica ante la realidad multicultural: análisis de la situación y propuestas de mejora. Revista Electrónica Interuniversitaria de Formación del Profesorado, 17(2), 183-199. Disponible en http://revistas.um.es/reifop/article/view/183

Hargreaves, A. (2005). Profesorado, cultura y postmodernidad: cambian los tiempos, cambia el profesorado. Madrid: Ediciones Morata. Disponible en http://www.terras.edu.ar/biblioteca/17/GSTN_Hargreaves_Unidad_2.pdf

Herrera, L. (2015). El cosmopolitanismo y la interculturalidad. Un análisis desde una contribución teórico-crítica. REALIS, Revista de Estudios AntiUtilitaristas e PosColoniais. 5(1), 203221. Disponible

en https://www.researchgate.net/profile/Luis_Herrera_Montero/publication/303658223_EL_C OSMOPOLITANISMO Y LA INTERCULTURALIDAD Un analisis desde una contr ibucion_teorico-critica/links/574̄bb28f08ae5c51e29eae86/EL-COSMOPOLITAN̄ISMO-Y-YLA-INTERCULTURALIDAD-Un-analisis-desde-una-contribucion-teorico-critica.pdf

Krainer, A., Aguirre, D., Guerra, M. y Meise, A. (2017). Educación superior intercultural y diálogo de saberes: el caso de la Amawtay Wasi en Ecuador. RESU, Revista de la Educación Superior, 46 (184), 55-76. https://doi.org/10.1016/j.resu.2017.11.002

Krainer, A. y Guerra, M. (2016). Interculturalidad y educación. Desafíos docentes. Ecuador: Flacso. Disponible en http://www.flacsoandes.edu.ec/libros/digital/56204.pdf

Lehmann, D. (2015). Convergencias y divergencias en la educación superior intercultural en México. Universidad Nacional Autónoma de México. Revista Mexicana de Ciencias Políticas y Sociales, Nueva Época, Año LX, 223, 133-170. Disponible en https:/www.sciencedirect.com/science/article/pii/S0185191815721331 
López, L. E. (2009). Interculturalidad, educación y política en América Latina: perspectivas desde el Sur: pistas para una investigación comprometida y dialogal. In L. E. López (Ed.) Interculturalidad, educación y ciudadana (pp. 129-217). La Paz: Plural/FUNPROEIB Andes.

Moya, R. (1998), Reformas educativas e interculturalidad en América Latina. Revista Iberoamericana de Educación, núm. 17, pp. 105-187. Disponible en https://centroderecursos.cultura.pe/sites/default/files/rb/pdf/Reformas_educativas_e_inte rculturalidad_en_America_Latina.pdf

Olivera-Rodríguez, $\overline{\text { I. }}$ (2014). ¿Desarrollo o bien vivir? Repensando la función social de la Universidad Intercultural desde el cuestionamiento al efecto educativo. Anthropologica. Año XXXII, 33, 179-207. https://dialnet.unirioja.es/servlet/articulo?codigo $=5039585$

Presidencia de la República. (31 de marzo de 2011). Ley Orgánica de Educación Intercultural. (LOEI). Registro Oficial No 417. Disponible en https://educacion.gob.ec/wpcontent/uploads/downloads/2017/05/Ley-Organica-Educacion-Intercultural-Codificado.pdf

Rodríguez, R. (2005). Estudio de las concepciones de estudiantes de magisterio sobre la diversidad cultural. $\quad$ Educar, 28, 46-69. Disponible en https://www.raco.cat/index.php/Educar/article/view/39742

Rodríguez, F. J. \& Hernández, P. J. (2011). Cuestionario de Actitudes hacia la MulticulturalidadCAM.

Disponible en http://www3.gobiernodecanarias.org/medusa/ecoescuela/tamadaba/2011/11/30/cuestionario -de-actitudes-hacia-la-multiculturalidad-cam/

Santos, M. A., Cernadas, F. X. \& Lorenzo, M. M. (2014). La inclusión educativa de la inmigración y la formación intercultural del profesorado. Revista Electrónica Interuniversitaria de Formación del Profesorado, 17(2), 123-137. Disponible en https://revistas.um.es/reifop/article/view/123

Speiser, S. (1999). El para qué de la educación en interculturalidad. En Ruth Moya (ed.). Interculturalidad y educación diálogo para la democracia en América Latina (pp.85-96). Quito: Abya-Yala

Treviño, E., Villalobos, C. \& Baeza, A. (2016). Recomendaciones de Políticas Educativas en América Latina en base al TERCE ha sido elaborado por encargo de la Oficina Regional de Educación para América Latina y el Caribe, Santiago de Chile: OREALC/ UNESCO. Disponible en https://www.gcedclearinghouse.org/resources/educational-policyrecommendations-based-terce?language $=\mathrm{es}$

Tubino, F. (2004). Del interculturalismo funcional al interculturalismocrítico. En Samaniego Mario y Garbarini Carmen, Rostros y Fronteras de la Identidad (pp. 151-165). Temuco: Universidad Católica de Temuco. Disponible en https://centroderecursos.cultura.pe/sites/default/files/rb/pdf/Del\%20interculturalismo\%2 0funcional $\% 20$ al $\% 20$ interculturalismo $\% 20$ critico.pdf

UNESCO (2016). ¿Qué afecta el aprendizaje de los niños migrantes? UNESCO. Oficina de Santiago. Oficina Regional de Educación para América Latina y el Caribe. TERCE. En la mira, 4.4 Disponible en http://repositorio.minedu.gob.pe/bitstream/handle/123456789/4553/que\%20afecta\%20el\%20 aprendizaje $\% 20 \mathrm{de} \% 2010 \mathrm{~s} \% 20 \mathrm{ni} \% \mathrm{C} 3 \% \mathrm{~B} 1$ os $\% 20$ migrantes.pdf?sequence $=1 \&$ isAllowed $=\mathrm{y}$ 
Uribe-Pérez, M. \& Mosquera, C. (2016). La formación del profesorado desde el enfoque intercultural. Una aproximación a su estado actual. Episteme y Didaxis. No. Extraordinario, 1698-1704. Disponible en http://revistas.pedagogica.edu.co/index.php/TED/article/download/4802/3933

Viaña, J. (2011). Reconceptualizando la interculturalidad. En J. Viaña, C. Walsh y L. Tapia, Construyendo interculturalidad crítica. La Paz: Instituto Internacional de Integración Convenio Andrés Bello -III-CAB-. Disponible en https://www.yumpu.com/es/document/read/12604172/descargar-instituto-internacionalde-integracion-convenio-andres- $/ 32$

Villagómez, R. Ma \& Cunha de Campos, R. (2014). Buen vivir y educación para la práctica de la interculturalidad en el Ecuador. Otras prácticas pedagógicas son necesarias. Alteridad. Revista de Educación, 9(1), enero-junio, 35-42. Disponible en https://alteridad.ups.edu.ec/index.php/alteridad/article/view/1.2014.03 